\title{
Forebrain Contribution to the Induction of a Cellular Correlate of Conditioned Taste Aversion in the Nucleus of the Solitary Tract
}

\author{
Glenn E. Schafe, Randy J. Seeley, and llene L. Bernstein \\ Department of Psychology, University of Washington, Seattle, Washington 98195
}

A conditioned taste aversion (CTA) is a form of classical conditioning in which animals avoid a taste (conditioned stimulus; CS) which has been previously paired with a treatment (unconditioned stimulus; US) that produces transient illness. Recently, a reliable cellular correlate of the behavioral expression of a CTA was identified using c-Fos immunostaining as a marker of neuronal activation. Exposure to a saccharin solution (CS) which had previously been palred with lithium chloride (LiCl; US) induced significant c-Fos-like immunoreactivity (c-FLI) in the intermediate zone of the nucleus of the solitary tract (NTS), a response that was quite similar to that displayed following administration of $\mathrm{LiCl}$ alone. The present studies employed a variant of the chronic decerebrate rat preparation to explore whether circuitry intrinsic to the brainstem is sufficient for the induction of c-FLI in NTS both as an unconditioned response to the $\mathrm{LiCl}$ and as a conditioned response to the saccharin. Using chronic hemidecerebrate rats, which have a unilateral brain transection at the level of the superior colliculus, we found that the unconditioned c-FLI to $\mathrm{LiCl}$ was unaltered by the transection, while the conditioned expression of C-FLI to the CS taste was evident only on the side of the NTS which retained neural connections with the forebrain. These findings strongly implicate forebrain input in this cellular correlate of CTA learning and also indicate that the pathways mediating the response to the US (LiCl) and the CS (saccharin) differ.

[Key words:c-Fos, decerebrate rats, learning, brainstem, lithium chloride, aversion conditioning]

Taste aversion learning is a form of classical conditioning in which animals avoid a taste (conditioned stimulus; CS) previously paired with a treatment (unconditioned stimulus; US) that produces transient illness (Garcia et al., 1974). The robustness and rapid acquisition of this response suggest that the critical underlying neural events are also robust and, hence, detectable using appropriate methodologies. However, despite extensive interest in taste aversion learning (Riley and Tuck, 1985), the conditioned taste aversion (CTA) paradigm has not been widely adopted as a model for studying the cellular events underlying associative lcarning and, hence, relatively little is known about the neural pathways that mediate it.

Received April 19, 1995; revised June 12, 1995; accepted June 14, 1995

This research was supported by NIH Grants DC00248, AA07455, DK17844, and a Royalty Research Grant from the University of Washington.

Correspondence should be addressed to Ilene L. Bernstein, University of Washington, Department of Psychology, 119 Guthrie Hall, Box 351525, Seattle, WA 98195-1525.

Copyright $(\odot 1995$ Society for Neuroscience $0270-6474 / 95 / 156789-08 \$ 05.00 / 0$
Despite the fact that brainstem regions such as the nucleus of the solitary tract (NTS) might appear to be sufficient for the integration of taste-illness associations (Hermann and Rogers, 1985) work with chronic decerebrate rats suggests otherwise. The chronic decerebrate rat fails to acquire CTAs despite multiple conditioning trials (Grill and Norgren, 1978a). Paradoxically, studies employing localized forebrain lesions do not point clearly to a specific forebrain location as necessary for CTA associations. Instead, lesions of structures such as gustatory neocortex (Lasiter and Glanzman, 1982; Kiefer et al., 1984) and basolateral amygdala (Simbayi et al., 1986) attenuate but do not eliminate CTA acquisition. Thus, although the decerebrate experiments point to forebrain input as critical to CTA learning, lesion studies fail to clearly identify the source of that input.

Using c-Fos-like immunoreactivity (c-FLI) as a marker of neuronal activation, we recently identified a cellular correlate of CTA expression (Swank and Bernstein, 1994). Exposure to a taste CS (saccharin) that had previously been paired with $\mathrm{LiCl}$ induced significant c-FLI in NTS, a pattern similar to that induced by the US drug. Significant c-FLI was not evident in controls exposed to saccharin, nor was it seen in rats displaying an innate aversion to quinine (Swank et al., 1995). Using a similar approach, Houpt et al. (1994) found that extinction of the behavioral CTA is associated with extinction of the expression of c-FLI. Thus, c-FLI in NTS after reexposure to a saccharin CS appears to be a reliable correlate of CTA expression.

The present studies employed a variant of the chronic decerebrate rat preparation, examining behavioral and c-FLI indices of CTA learning in rats with unilateral supracollicular brain transections. This approach offered a number of advantages over the fully transected decerebrate. First, it avoided the ambiguity of interpreting negative behavioral data in animals whose behavior is seriously compromised. When the behavior is no longer the primary dependent measure, the role of the forebrain in CTA learning can be addressed in a far less compromised animal. In addition, it became possible to use the level of c-FLI on the side of the brain contralateral to the transection as a control. This is the first report, of which we are aware, to make use of a chronic hemidecerebrate rat preparation. Combining hemidecerebration with c-Fos immunostaining provided clear and unambiguous evidence for a forebrain contribution to the responsc to the CS (taste) but not the US ( $\mathrm{LiCl})$, and demonstrates the potential of this combination of techniques to reveal critical functional interactions between forebrain and brainstem.

\section{Materials and Methods}

Subjects. Forty adult, male Long-Evans rats, weighing between 300 and $400 \mathrm{gm}$, were obtained from the breeding colony at the University of Washington. Rats were housed individually in suspended stainless steel 
cages and maintained on a 12:12 hr light:dark cycle. Teklad rodent chow and water wcre provided ad libitum, unless otherwise indicated.

\section{Experiment 1: examination of patterns of c-FII in the brainstem of hemidecerebrate rats following administration of $\mathrm{LiCl}$ or saline}

Brainstem transections. Under Equithesin $(3.3 \mathrm{mg} / \mathrm{kg})$ or a mixture of Ketamine $(86 \mathrm{mg} / \mathrm{kg})$ and Xylazine $(12.9 \mathrm{mg} / \mathrm{kg})$ anesthesia, rats were given either hemidecerebrate transections (HEMI; $n=8$ ) or SHAM operations $(n=8)$. Briefly, a $1.0 \mathrm{~mm}$ opening in the frontal plane was drilled into the skull, either right or left of the midline, $60 \%$ of the distance between bregma and lambda. A $0.5 \mathrm{~mm}$ wide, blunt L-shaped spatula was then inserted just lateral to the midsagittal sinus and gently advanced to the cranial floor. The spatula was stepped in small increments to the most lateral point on either the right or left side. The side of the transection (right or left) was counterbalanced within groups. For SHAM animals, the skull was opened and the dura cut, but no transection was made.

Rats were given $4 \mathrm{~d}$ to recover following surgery. Due to hypophagia, hemidecerebrate animals were supplemented with intragastric intubations of sweetened condensed milk diet and water in a 1:1 mixture. Intubations of $6 \mathrm{ml}$ were performed three times daily throughout the remainder of the experiment. Weights were taken every other day to assess recovery.

Drug administration. Five days following surgery, rats in each of the two surgical groups (HEMI; SHAM) were weight matched and assigned to one of two treatment groups that received an intraperitoneal (i.p.) injection of either $\mathrm{LiCl}(0.15 \mathrm{M} ; 20 \mathrm{ml} / \mathrm{kg})$ or an equivalent volume of isotonic saline $(\mathrm{Na})$. This yielded the following experimental groups: SHAM-Li $(n=4)$; SHAM-Na $(n=4)$, HEMI-Li $(n=4)$, and HEMINa $(n=4)$. Two hours following injection, rats were given an overdose of pentobarbital anesthetic $(80 \mathrm{mg} / \mathrm{kg})$ and transcardially perfused with isotonic phosphate-buffered saline (PBS) followed by $4 \%$ paraformaldehyde in $0.1 \mathrm{M}$ phosphate buffer. Brains were removed from the skull for immunohistochemical processing.

c-Fos immunohistochemistry. Following a $24-48 \mathrm{hr}$ postfixation in $4 \%$ paraformaldehyde, the caudal brainstem was blocked and prepared for sectioning. The remaining forebrains were immersed in a solution of $10 \%$ buffered formalin $/ 15 \%$ sucrose for later histological processing. Brainstem sections of $50 \mu$ thickness were made in the horizontal plane, thus allowing visualization of the entire rostro-caudal extent of the NTS as well as the pontine parabrachial nuclei $(\mathrm{PBN})$. Slices were rinsed ( $3 \times, \mathrm{PBS}$ ), incubated for $20 \mathrm{~min}$ in $0.3 \% \mathrm{H}_{2} \mathrm{O}_{2}$ in absolute methanol to quench endogenous peroxidase, rinsed ( $3 \times, \mathrm{PBS})$, and incubated for 1 $\mathrm{hr}$ in $1 \%$ gelatin, $5 \%$ normal goat serum in PBS. Slices were then transferred without rinsing to the primary antibody solution, which consisted of $0.1 \mu \mathrm{g} / \mathrm{ml}$ polyclonal rabbit antiserum (Oncogene Science, Uniondale, NY), which recognizes residues $4-17$ of the Fos protein. After a $36 \mathrm{hr}$ incubation at $4^{\circ} \mathrm{C}$, slices were rinsed $(10 \times, \mathrm{PBS}, 2 \mathrm{hr})$ and processed using the $\mathrm{ABC}$ method (Vector Laboratories, Burlingame, CA). Briefly, slices were transferred to biotinylated anti-rabbit antibody for $1 \mathrm{hr}$, rinsed $(10 \times$, PBS; $1 \mathrm{hr})$, transferred to avidin-biotin peroxidase for $1 \mathrm{hr}$, rinsed (5×, PBS, $30 \mathrm{~min}$, then $5 \times$ PBS, $30 \mathrm{~min}$ ), and developed with diaminobenzidine substrate (4 min). Following development, slices were rinsed (10×, PBS, $30 \mathrm{~min}$ ), mounted on glass slides, and coverslipped with Permount.

Scoring of cell nuclei positive for $c$-FLI. Preliminary observations revealed that $c$-FLI was present in NTS, specifically those regions that circumscribed the area postrema (AP), and also sections extending less than $200 \mu$ ventral to the plane of the AP. Camera lucida drawings from sections that included the NTS, PBN, AP, and others ventral to this region were prepared and examined by observers blind to experimental conditions. Templates were then scored, and the number and location of cell nuclei positive for c-FLI in NTS, PBN, and AP was recorded. Scores across raters were averaged for statistical analysis with ANOVAs and paired $t$ tests.

Forebrain histology. To verify the distance of transections from the midline as well as overall location and completeness in the rostro-caudal plane, $50 \mu$ sections from the forebrains of hemidecerebrate animals were cut in the horizontal plane using a Cryostat. Every fourth section was thaw mounted onto gelatin-coated glass slides. Sections were air dried, stained for Nissl using thionin, dehydrated in ethanols, cleared in Histoclear, and coverslipped using Permount. Camera lucida drawings of sections were prepared and examined using a projection light micro- scope. Sections were analyzed by comparing them with those found in Paxinos and Watson (1986).

\section{Experiment 2: examination of patterns of c-FLI in the brainstem of hemidecerebrate rats during behavioral expression of a CTA}

Surgery. Using a Ketamine $(86 \mathrm{mg} / \mathrm{kg}$ ) and Xylazine $(12.9 \mathrm{mg} / \mathrm{kg}) \mathrm{mix}-$ ture, rats were anesthetized and implanted with bilateral intraoral cannulae and given either hemi-transections (HEMI; $n=8$ ) or SHAM operations $(n=8)$. Oral cannulas constructed of PE-50 tubing were inserted with a 23-gauge sharpened stainless steel probe. The probe was inserted through the roof of the mouth just rostro-lateral to the first molar, and passed through the cheek, caudal to the eye, to exit the scapular area behind the head. Following cannula placement, hemi-transections wcre performed as described above. The side of the transection (right or left) was counterbalanced within groups. At the time of surgery, rats received $0.2 \mathrm{cc}$ Bicillin (penicillin $\mathrm{G}$ benzathine, 300,000 $\mathrm{U} / \mathrm{ml}, \mathrm{i} . \mathrm{m}$.) as a prophylaxis against infection, and each rat was provided with Teklad powdered rodent chow for the remainder of the study. Weights were taken every other day to assess recovery.

Flushing and habituation. Rats were given $5 \mathrm{~d}$ to recover following surgery, during which time oral cannulae were flushed daily with distilled water to prevent clogging. Hemidecerebrates were supplemented with $8 \mathrm{ml} \mathrm{1:1}$ sweetened condensed milk and water three times daily. Two days prior to conditioning, rats were habituated to plastic cylindrical chambers that were to be used during conditioning. On each habituation day, rats were placed in a cylinder for $30 \mathrm{~min}$ and infused by infusion pump with $5 \mathrm{ml}$ distilled water at a rate of $0.5 \mathrm{ml} / \mathrm{min}$. Rats in each of the two surgical groups (HEMIS; SHAMS) were weight matched and assigned to one of two conditioning groups that differed in terms of the drug they would receive during conditioning $(\mathrm{LiCl}$ or saline). This yielded the following experimental groups: SHAM-paired $(n=4)$; SHAM-unpaired $(n=4)$; HEMI-paired $(n=4)$; and HEMIunpaired $(n=4)$.

Conditioning. Each rat received two conditioning trials, each of which consisted of a $3 \mathrm{~d}$ cycle. On the first day of each cycle, rats were placed in the experimental chamber and infused with $5 \mathrm{ml}$ of $0.15 \%$ saccharin at a rate of $0.5 \mathrm{ml} / \mathrm{min}$. Infusions were videotaped to later assess the time at which any rejection responses, such as passive drip from the mouth, occurred. Rejection latencies were determined by the time elapsing between onset of the intraoral infusion and the onset of persistent, passive dripping of the solution from the oral cavity. Immediatcly following the infusion period, paired animals received an injection of $0.15 \mathrm{M} \mathrm{LiCl}(20 \mathrm{ml} / \mathrm{kg}$, i.p.). Unpaired animals were injected with an equivalent volume of $0.15 \mathrm{M} \mathrm{NaCl}$.

On the following day paired animals received noncontingent injections of $0.15 \mathrm{M} \mathrm{NaCl}$, while unpaired animals received noncontingent injections of $0.15 \mathrm{M} \mathrm{LiCl}$. This was done to ensure that all rats had equivalent exposure to both the CS solution and lithium, the only difference being whether the two stimuli were paired. The third day served as a recovery day with rats maintained on their regular food and water regimen and given no injections.

Testing. On the test day, rats were placed in the experimental chamber and reexposed to the CS taste $(5 \mathrm{ml} ; 0.5 \mathrm{ml} / \mathrm{min})$. As during conditioning, animals were videotaped for subsequent analysis of time until solution rejection. Following saccharin infusion, rats were returned to their home cage. Two hours following saccharin exposure, rats were sacrificed. Brains were removed and brainstems were processed for c-FLI. Forebrain histology was performed to assess location and extent of transections.

\section{Results}

\section{Experiment 1: c-FLI to US drug in the hemidecerebrate}

Mean number of cell nuclei positive for c-FLI for both SHAM and HEMI animals in AP, lateral PBN, and intermediate NTS is presented in Figures 1, 2, and 3, respectively. Sample photomicrographs through intermediate NTS can be viewed in Figure 4. Counts for lateral PBN and intermediate NTS represent cell nuclci ipsilatcral and contralateral to the transection. Analysis of the data revealed that $\mathrm{LiCl}$ induced significantly more $\mathrm{c}-\mathrm{FLI}$ in all structures examined relative to saline-injected controls [AP, $F(1,12)=145.2, p<0.001 ;$ NTS, $F(1,12)=47.2, p<0.001 ;$ 


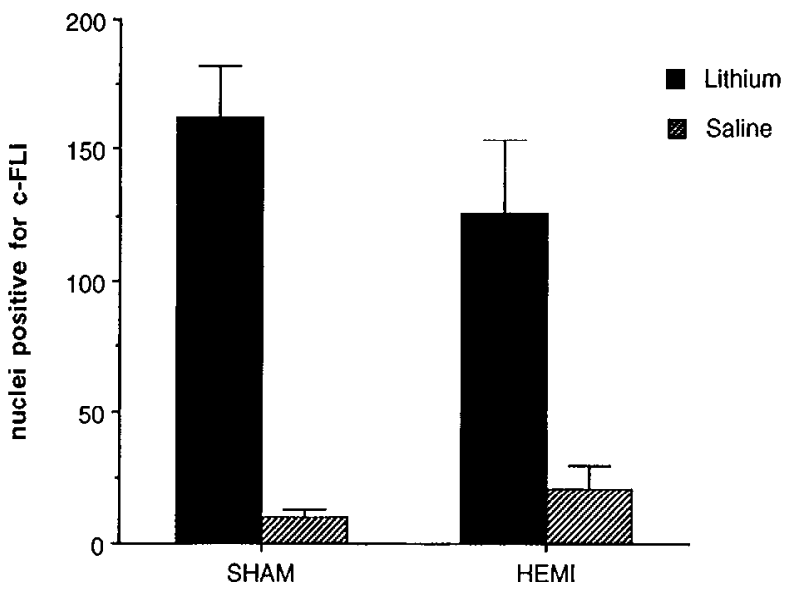

Figure 1. Mean number $( \pm \mathrm{SE})$ of nuclei positive for c-FLI in the area postrema (AP) of SHAM and HEMI animals following i.p. injection of $\mathrm{LiCl}$ (black bars) or $\mathrm{NaCl}$ (striped bars).

PBN, $F(1,12)=83.8, p<0.001]$, and no differences were detected between ipsilateral and contralateral sides. Although overall induction of c-FLI in HEMI animals appeared somewhat lower than that in SHAM animals, this difference was not statistically significant. Thus, the data indicate that the pattern of c-FLI in structures within the brainstem of both SHAM and HEMI animals is comparable following $\mathrm{LiCl}$ treatment, and that hemi-
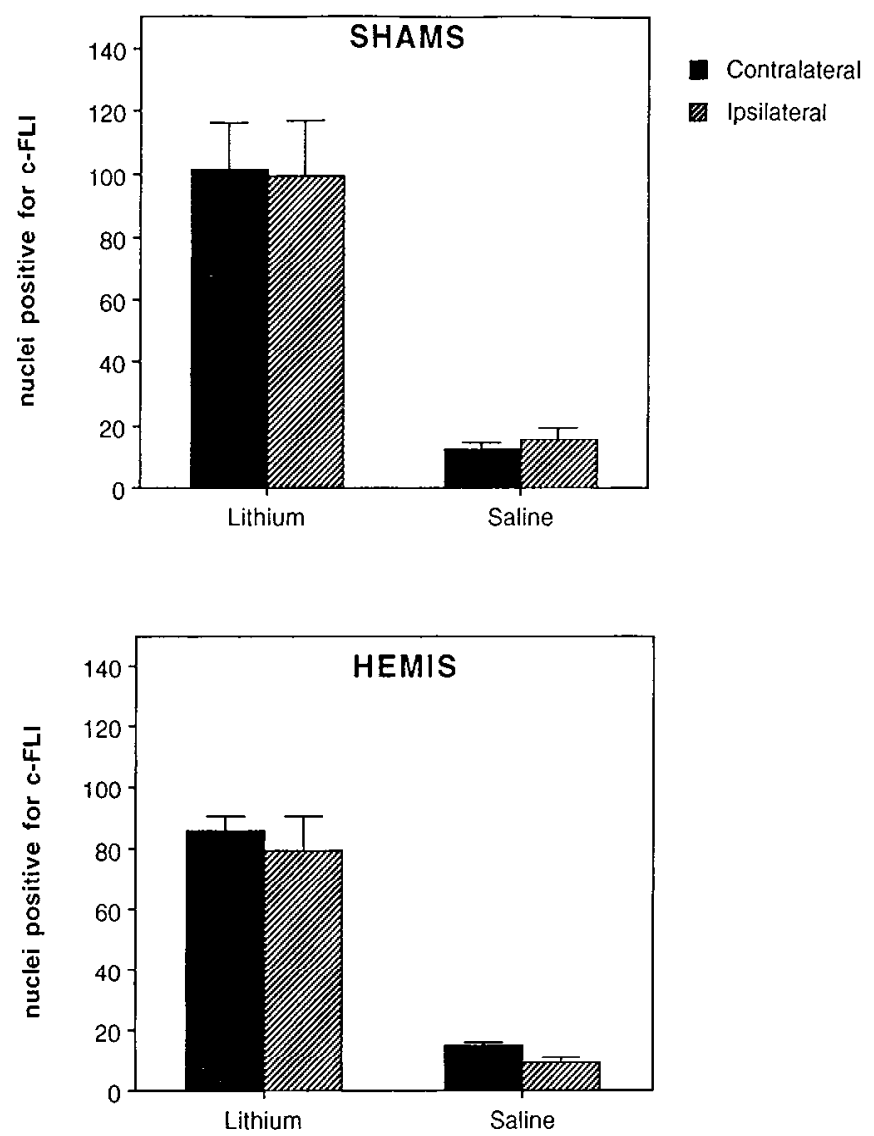

Figure 2. Mean number $( \pm \mathrm{SE})$ of nuceli positive for c-FLI in the pontine parabrachial nucleus (PBN) of SHAM (top) and HEMI (bottom) animals following $\mathrm{LiCl}$ or $\mathrm{NaCl}$. Black bars represent the intact (contralateral to the transection) and striped bars the cut side (ipsilateral to the transection).
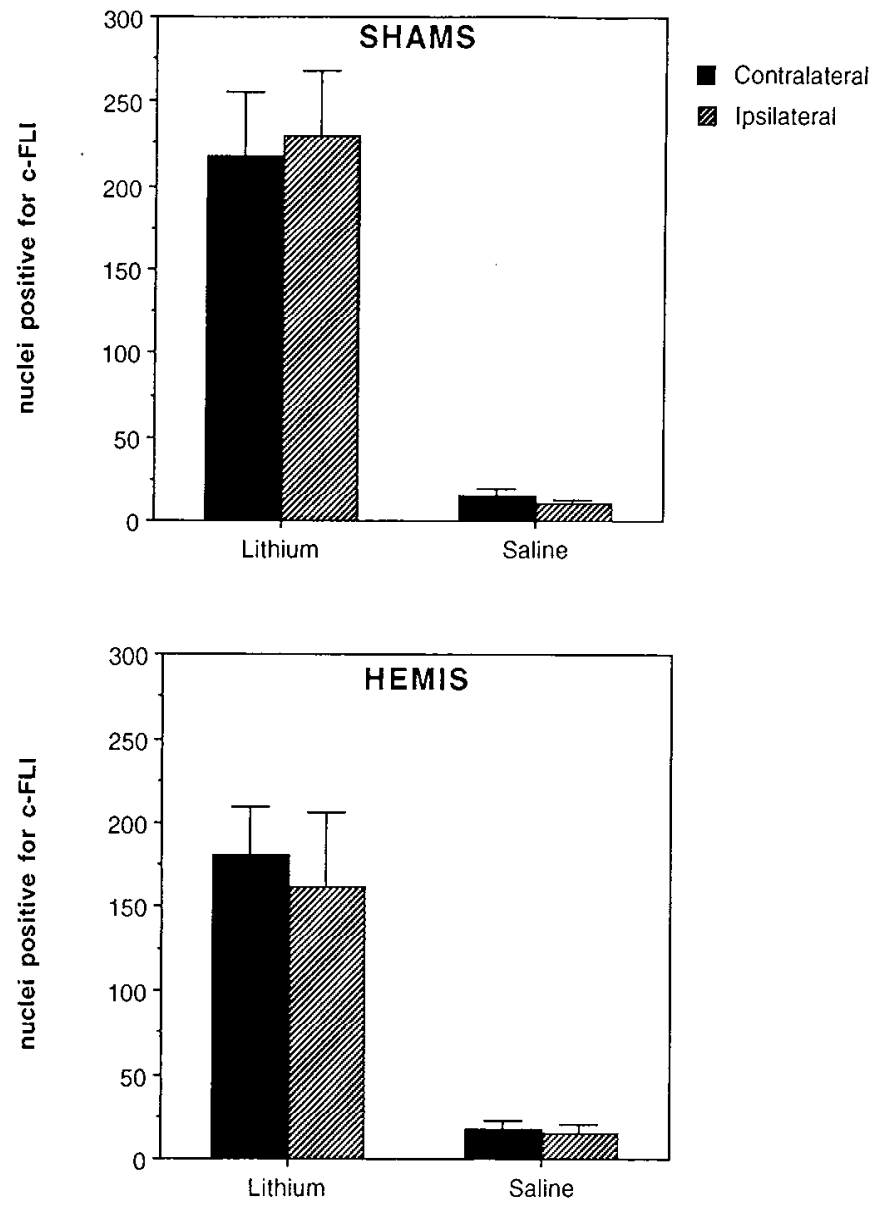

Figure 3. Mean number $( \pm \mathrm{SE})$ of nuceli positive for c-FLI in the intermediate division of the nucleus of the solitary tract (iNTS) of SHAM (top) and HEMI (bottom) animals following i.p. injection of either $\mathrm{LiCl}$ or $\mathrm{NaCl}$. Black bars represent the intact side (contralateral to the transection) and striped bars represent the cut side (ipsilateral to the transection).

decerebration does not alter the expression of c-FLI ipsilateral to the transection either in $\mathrm{LiCl}$ - or saline-treated animals.

\section{Experiment 2: c-FLI during expression of a CTA in the hemidecerebrate}

Mean latency to reject the intraoral infusion of CS saccharin following conditioning is presented in Figure 5, while mean number of cell nuclei positive for c-FLI in intermediate NTS of the same animals is presented in Figure 6. Sample photomicrographs may be viewed in Figure 7 . Following two saccharin$\mathrm{LiCl}$ pairings, both SHAM and HEMI animals rejected the saccharin within 1-4 min, while continued ingestion of the saccharin was evident in unpaired controls. Mann-Whitney $U$ tests determined that the effects of drug pairing on time to reject were significant (SHAM-paired vs SHAM-unpaired, $p<0.01$; HEMIpaired vs HEMI-unpaired, $p<0.01$ ). Mean rejection latency in SHAM-paired versus HEMI-paired animals was also found to be different, but the extent to which this difference can be altributed to neurological impairment or to the transected animals' prior exposure to sweetened condensed milk remains unclear.

In SHAM animals, the saccharin CS induced a significant bilaterally symmetrical pattern of c-FLI in NTS, while little to no staining was evident in unpaired controls $[F(1,6)=53.0, p$ $<0.001]$. In HEMI-paired animals, however, the pattern of 

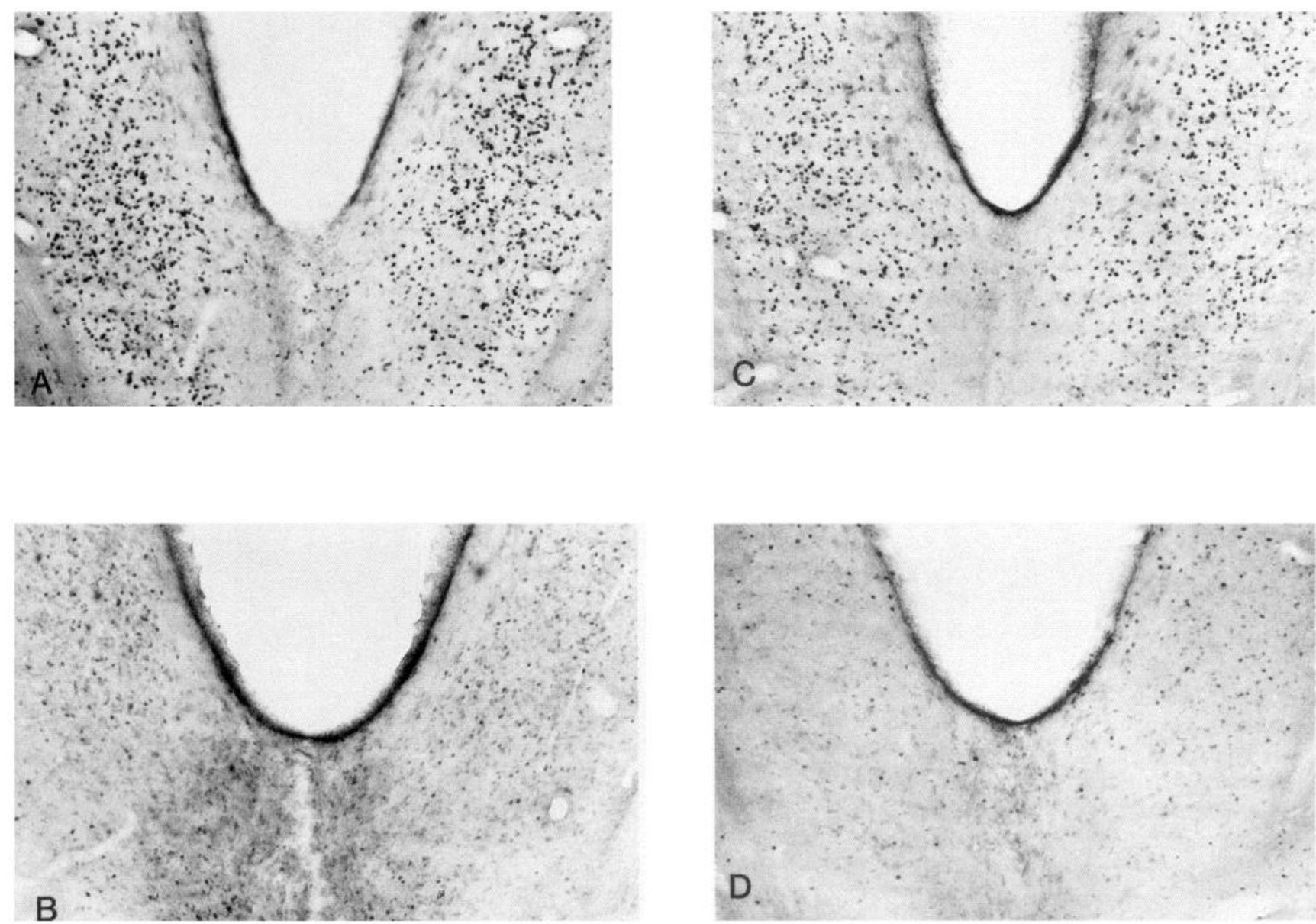

Figure 4. Photomicrographs of horizontal sections through the brainstem encompassing the NTS, AP, and fourth ventricle. The sections are from representative animals in SHAM $[\mathrm{LiCl}(A) ; \mathrm{NaCl}(B)]$ and $\mathrm{HEMI}[\mathrm{LiCl}(C) ; \mathrm{NaCl}(D)]$ groups following administration of the US drug. In $\mathrm{HEMI}$ animals, the transection is on the right side.

c-FLI following CS reexposure was quite asymmetrical. In paired animals, expression of c-FLI was evident contralateral to the transection, while significantly less expression was evident on the ipsilateral side $[F(1,6)=225.3, p<0.001]$. In fact, the number of cells expressing c-FLI ipsilateral to the transection in HEMI-paired animals was not found to be significantly different from that of unpaired controls. Consistent with our previous

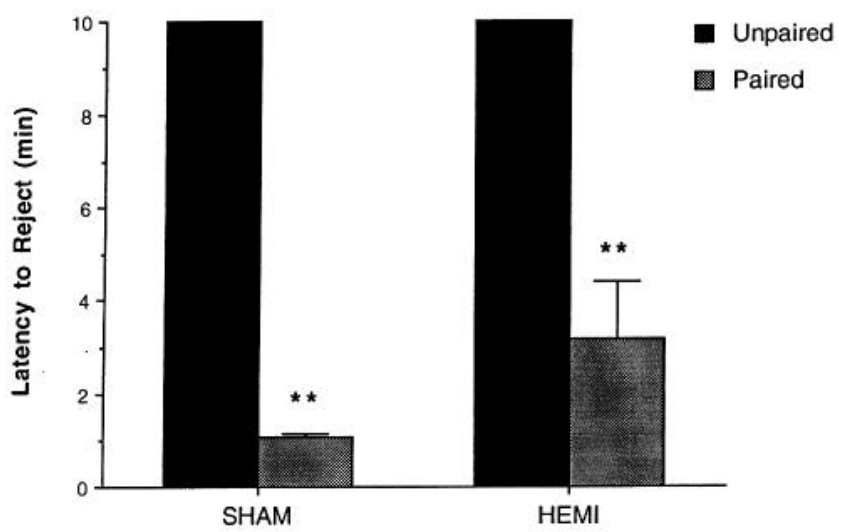

Figure 5. Mean rejection latency ( \pm SE) of SHAM and HEMI animals during intraoral saccharin infusion at the time of testing. Saccharin had either been paired (gray bars) or unpaired (black bars) with LiCl. ${ }^{* *} p$ $<0.01$ paired relative to unpaired. work (Swank and Bernstein, 1994; Swank, Schafe and Bernstein, 1995), the distribution of the majority of cells expressing c-FLI following CS reexposure appeared to be confined to the medial subnucleus of the "intermediate" division of NTS (Barraco et al., 1992), a region that lies just rostral to the obex and receives both gustatory and visceral projections (Hamilton and Norgren, 1984). No increases in c-FLI were detected in PBN or AP following CS reexposure.

Based on the bilaterally symmetrical expression of c-FLI to $\mathrm{LiCl}$, we do not believe that the marked asymmetry of the conditioned c-FLI response in hemidecerebrates is due to a paucity of cells on the side of the NTS ipsilateral to the transection consequent to retrograde degeneration. However, since the delay interval between transection and sacrifice differed in these two studies ( $5 \mathrm{~d}$ for the $\mathrm{LiCl} ; 10 \mathrm{~d}$ for the taste), we injected another group of hemidecerebrates with $\mathrm{LiCl} 10 \mathrm{~d}$ after transection. $\mathrm{Re}$ sults indicated an essentially identical, symmetrical pattern of c-FLI in NTS to that seen in the first experiment (mean \pm SE c-FLI positive cells: ipsilateral to transection $=268.7 \pm 21.2$; contralateral to transection $=281.3 \pm 4.3$ ). Thus, neural connections with the forebrain appear to be necessary for the induction of c-FLI in NTS during expression of a CTA but not for the response to the US drug, $\mathrm{LiCl}$.

\section{Forebrain histology}

A representative forebrain section of a hemidecerebrate animal at the level of the anterior commissure is presented in Figure 8. 

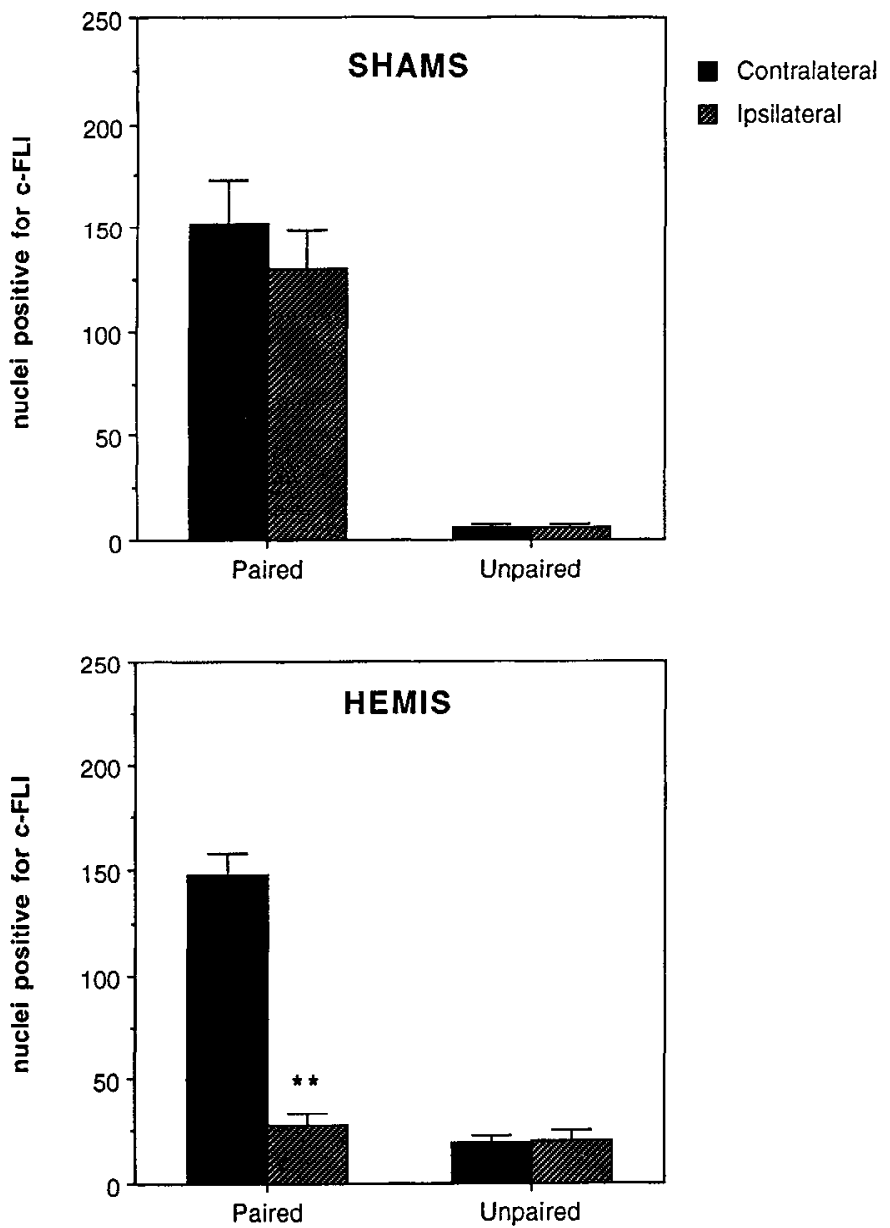

Figure 6. Mean number $( \pm S E)$ of nuclei positive for c-FLI in intermediate division of the nucleus of the solitary tract (iN'S) of SHAM (top) and HEMI (bottom) animals following exposure to saccharin previously paired or unpaired with $\mathrm{LiCl}$. Black bars represent the intact side (contralateral to the transection) and striped bars represent the cut side (ipsilateral to the transection). ${ }^{* *}$ Significant at $p<0.01$ ipsilateral relative to contralateral.

Examination of sections revealed that all animals had complete transections extending between 0.258 and $1.367 \mathrm{~mm}$ from the midline, with a mean distance of $0.759 \mathrm{~mm}(\mathrm{SD}=0.385)$. The rostro-caudal location of the transection was determined by comparing each section with representatives found in Paxinos and Watson (1986) and expressing the location as a percentage of the distance between bregma and lambda. Using this method, all transections were found to lie within $50-60 \%$ of this distance, just caudal to the thalamus and rostral to the superior colliculus.

\section{Discussion}

$\mathrm{LiCl}$, a toxin frequently employed as US in CTA studies, induces c-FLI in NTS and PBN, two brainstem areas that also receive convergent taste input. Following a single pairing of a saccharin solution with $\mathrm{LiCl}$, conditioned animals, but not controls, display a pattern of c-FLI in NTS that is similar to that produced by $\mathrm{LiCl}$, the US drug. Thus, after CTA training, reexposure to a taste CS induces a pattern of c-FLI within the NTS (but not PBN) which is not seen in the absence of conditioning (Swank and Bernstein, 1994). This response represents a striking correlate of CTA expression that provides a powerful tool for probing the neural mechanisms involved in this type of learning. The present studies demonstrate that induction of this cellular correlate of the behavioral expression of a CTA is dependent upon neural connections with the forebrain, since it is eliminated unilaterally by hemi-transection at the level of the superior colliculus. Since the expression of c-FLI to $\mathrm{LiCl}$ is unaltered by the same transection, we conclude that the pathways mediating the response to the drug (US) and the taste (CS) differ.

Grill and Norgren (1978a) reported that chronic decerebrate rats with bilateral transections showed no behavioral evidence of having acquired a CTA, despite multiple conditioning trials. In contrast, the hemidecerebrate rats in the present study developed significant aversions to the saccharin CS after two $\mathrm{LiCl}$ pairings. Thus, intact unilateral connections between forebrain and brainstem are sufficient to support acquisition and expression of a CTA. A more interesting observation addressed by these studies regards the role of forebrain connections in this type of learning. The key observation relevant to this issue is the observation that the neural correlate of conditioning, c-FLI in NTS, showed a distinct unilateral pattern of expression, with increases in c-FLI evident only on the side of the brainstem that retained neural connections with the forebrain. These findings support and extend those of Grill and Norgren (1978a) in indicating that the failure of the total decerebrate to display signs of CTA learning is not merely a reflection of impaired behavioral output.

The c-FLI response to the CS taste reflects an altered pattern of neuronal activation as a result of learning since it is not seen in response to exposure to saccharin unpaired with $\mathrm{LiCl}$ or to an innatcly aversive tastc, quininc (Swank et al., 1995). The region displaying c-FLI to the taste appears to represent a more restricted, more rostral subset of NTS cells than those that respond to $\mathrm{LiCl}$. While $\mathrm{LiCl}$ induces c-FLI in all rostro-caudal divisions medial to the solitary tract, the response to the CS taste appears to be restricted to the "intermediate" division of NTS (Barraco et al., 1992), a region that receives both taste and visceral projections (Hamilton and Norgren, 1984). This pattern of expression would be consistent with the interpretation that activation of these cells reflects feedback from cardiovascular or visceral reactions to the taste. The present results, however, make this interpretation unlikely since, despite clear behavioral rejection of the saccharin, in hemidecerebrate rats c-FLI in NTS was distinctly unilateral, while feedback from peripheral responses would be expected to appear bilaterally. In fact the unilateral transection approach reduces a frequent interpretative problem in marker studies of distinguishing neural changes due to behavioral response production from those more confined to mechanisms of plasticity. That connections with forebrain are necessary for the induction of the neural response supports the notion that activation of these NTS cells is a marker of plastic changes generated in the forebrain.

The utilization of the CTA paradigm as a model for studying cellular mechanisms of plasticity requires clear definition of the neural pathways involved in acquisition and expression of this learning. The pathways conveying information about the CS taste are well defined; involving gustatory nerves that terminate in the rostral division of NTS (Hamilton and Norgren, 1984; Finger, 1987). The pathways conveying information about the US drug are also known; including visceral information conveyed to the CNS by the vagus nerve as well as central detection within the AP (Borison, 1989; Bernstein et al., 1992; Koga and Fukuda, 1992). Thus, both CS and US activate brainstem areas, and brainstem transections involved in decerebration do not in- 

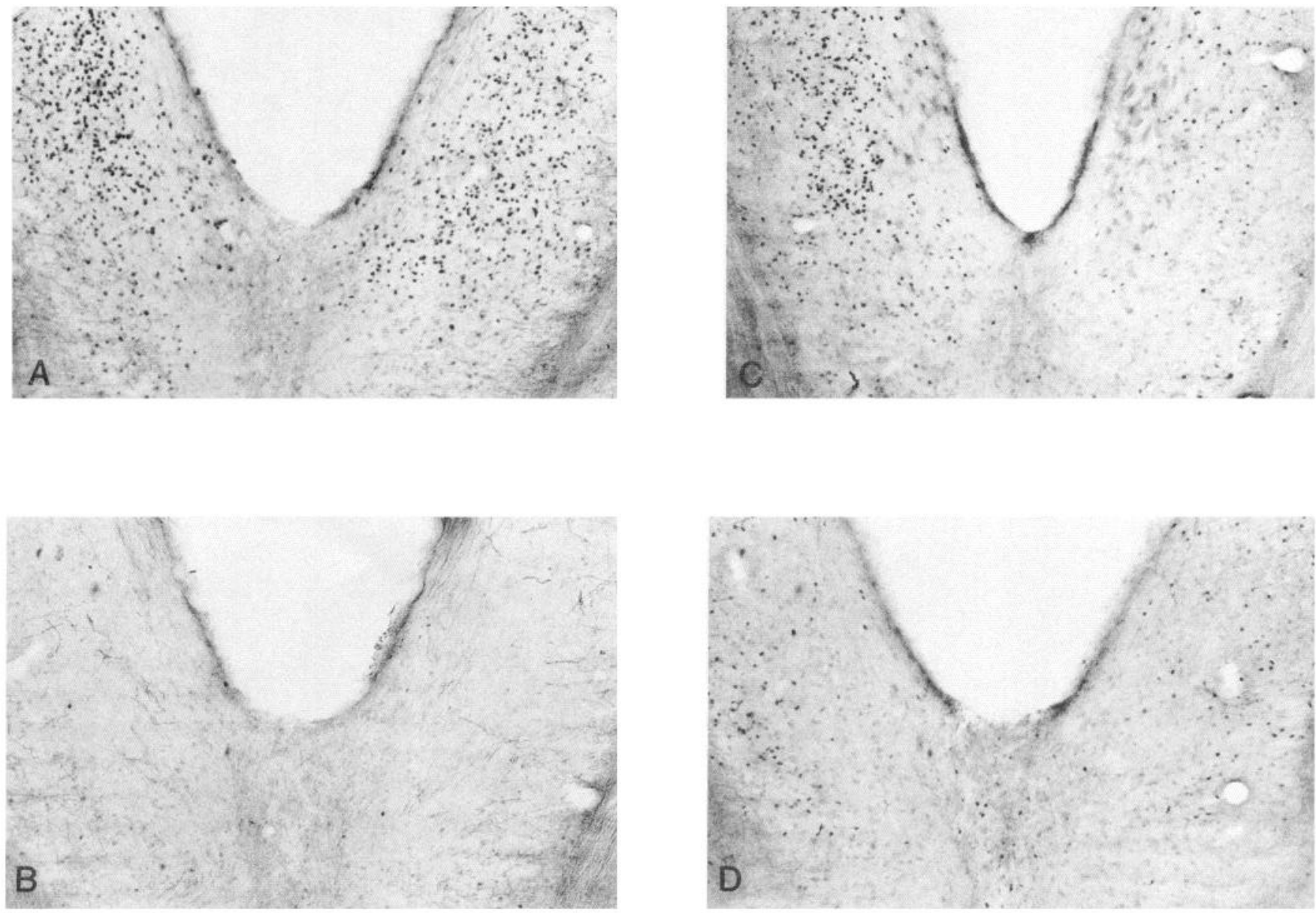

Figure 7. Photomicrographs of horizontal sections through the brainstem encompassing the NTS, AP, and fourth ventricle. The sections are from representative animals in SHAM [paired $(A)$; unpaired $(B)$ ] and HEMI [paired $(C)$; unpaired $(D)$ ] groups following CS reexposure. In HEMI animals, the transection is on the right side.

terfere with this activation (Hayama et al., 1985). Gustatory and visceral information converge not only in the NTS but also in the pontine PBN (Herman and Rogers, 1985). Recent studies provide convincing evidence that the PBN plays a critical role in CTA acquisition. Lesions of the medial or "gustatory" zone of PBN have been shown to completely block CTA acquisition (Spector et al., 1992) a deficit that appears due to a selective disruption of mechanisms responsible for taste-illness associations (Reilly et al., 1993). Interestingly, with the exception of $\mathrm{PBN}$, no other brain region has been demonstrated, unambiguously to be necessary for CTA acquisition.

It appears, then, that while the PBN is necessary for CTA acquisition, the decerebrate data indicate that it is not sufficient. This returns us to a consideration of potential forebrain sites that play a critical role in CTA learning in general, and the c-FLI correlate of this learning in particular. Neuroanatomical evidence indicates that virtually all forebrain structures that receive afferent input from NTS have descending projections back to it (Vangiersbergen et al., 1992). Prime candidates include insular cortex and central amygdaloid nucleus, as well as hypothalamic paraventricular and arcuate nuclei. The unilateral nature of the expression of c-FLI in hemidecerebrates excludes crossed connections within the NTS or below the level of the cut, but descending pathways that cross rostral to the cut cannot be excluded by the present data. Although there was considerable variation among the hemidecerebrates in the present study with regard to the proximity of the cut to the midline, there was very little variability in the dependent measure of c-FLI and no relationship between this measure and extent of the transection. Thus, although this analysis does not provide definitive information as to whether critical pathways lie along the midline, it does suggest that the forebrain structures and pathways mediating induction of c-FLI in NTS occupy a more lateral position.

Although the chronic decerebrate is thought to reflect the capacities of the isolated brainstem in integrating behaviors, the effect of the transection on structures caudal to the cut have not been well defined. The possibility exists that retrograde cellular degeneration occurs in regions such as the NTS and PBN which share extensive reciprocal connections with the forebrain. Thus, the failure of chronic decerebrates to acquire CTAs may not reflect direct forebrain involvement, but rather retrograde degeneration within a critical region such as PBN, which renders it nonfunctional. We do not consider this a likely explanation of the decerebrate data for two reasons. For one, in the present study, the number of cells in PBN displaying c-FLI in response to $\mathrm{LiCl}$ on the side of the brain ipsilateral to the transection was no different from that on the contralateral side. This strongly implies that cells responsive to $\mathrm{LiCl}$ are present and functional in comparable numbers on both sides of the brain after transection. Secondly, decerebration and PBN lesions generate different 

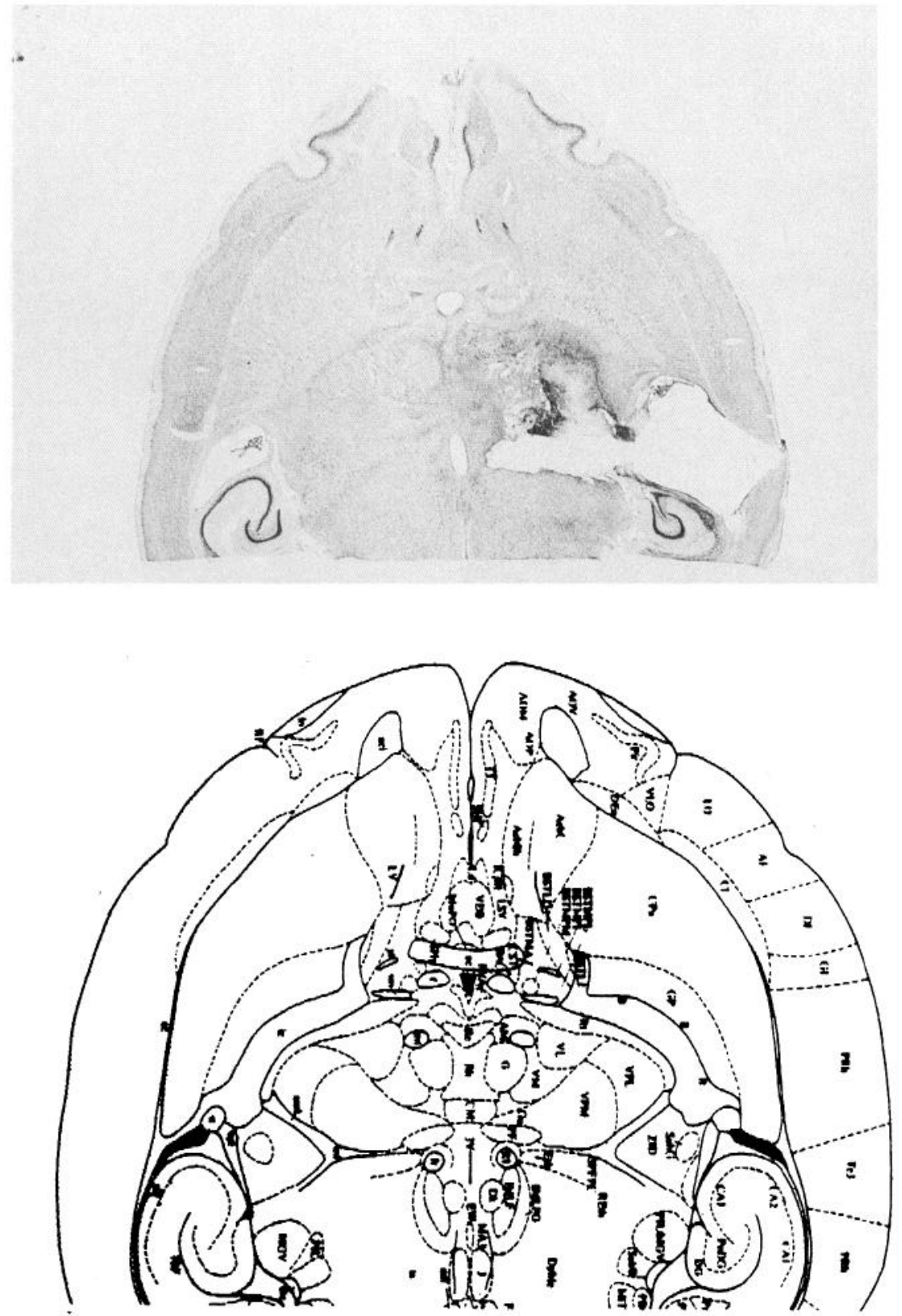

Figure 8. Photomicrograph of a horizontal section through the forebrain of a representative hemidecerebrate animal at the level of the anterior commissure. This section corresponds to the adjacent section adapted from Paxinos and Watson (1986). patterns of behavioral deficits in CTA learning. PBN lesions eliminate acquisition but not expression of CTAs, while decerebrates can neither acquire a new CTA nor express a previously acquired one (Grill and Norgren, 1978a; Reilly et al., 1993). In the present study, since animals were trained and tested after transection, it is unclear whether the absence of c-FLI in NTS reflects a deficit in acquisition or expression.

While supporting the conclusions of Grill and Norgren (1978a) that circuitry intrinsic to the brainstem is insufficient to support the acquisition and/or expression of a CTA, the present studies leave the identity of critical sources of input still undefined. The expanded use of the hemitransection method to explore the nature of this input offers a powerful technique for localizing key structures and pathways and determining whether their involvement can best be characterized as a localized or distributed network. The combination of unilateral transections and c-Fos immunohistochemistry provides a unique method for further exploring functional interactions between forebrain and brainstem in the expression of CTAs as well as other neuronal functions that cannot readily be isolated to specific brain regions.

\section{References}

Barraco R, El-Ridi M, Parizon EM, Bradley D (1992) An atlas of the rat subpostremal nucleus tractus solitarius. Brain Res Bull 29:703765.

Bernstein IL (1991) Flavor aversion. In: Smell and taste in health and disease (Getchell TV, Doty RL, Bartoshuk LM, Snow JB, eds), pp 417-428. New York: Raven.

Bernstein IL, Chavez M, Allen D, Taylor EM (1992) Area postrema mediation of physiological and behavioral effects of lithium chloride in the rat. Brain Res 575:132-137.

Borison HL (1989) Area postrema: chemoreceptor circumventricular organ of the medulla oblongata. Prog Neurobiol 32:351-390.

Chambers KC (1990) A neural model for conditioned taste aversions. Ann Rev Neurosci 13:373-385.

Finger TE (1987) Gustatory nuclei and pathways in the central nervous 
system. In: Neurobiology of taste and smell (Finger TE, Silver WL, eds), pp 331-354. New York: Wiley.

Garcia J, Hankins WG, Rusiniak KW (1974) Behavioral regulation of the milieu interne in man and rat. Science 185:824-831.

Grill HJ, Norgren R (1978a) Chronically decerebrate rats demonstrate satiation but not bait shyness. Science 201:267-269.

Grill HJ, Norgren R (1978b) The taste reactivity test: II: Mimetic responses to gustatory stimuli in chronic thalamic and chronic decerebrate rats. Brain Res 143:281-297.

Hamilton RB, Norgren R (1984) Central projections fo gustatory nerves in the rat. J Comp Neurol 222:560-577.

Hayama T, Ito S, Ogawa H (1985) Responses of solitary tract nucleus neurons to taste and mechanical stimulations of the oral cavity in decerebrate rats. Exp Brain Res 60:235-242.

Hermann GE, Rogers RC (1985) Convergence of vagal and afferent gustatory input within the parabrachial nucleus of the rat. $J$ Autonom Nerv Syst 13:1-17.

Houpt TA, Philopena JM, Wessel TC, Joh TH, Smith GP (1994) Increased c-fos expression in nucleus of the solitary tract correlated with conditioned taste aversion to sucrose in rats. Neurosci Lett 172: $1-5$.

Kiefer SW, Cabral RJ, Garcia J (1984) Neonatal ablations of the gustatory neocortex in the rat: taste aversion learning and taste reactivty. Behav Neurosci 98:804-812.

Koga T, Fukuda $H$ (1992) Neurons in the nucleus of the solitary tract mediating inputs from emetic vagal afferents and the area postrema to the pattern generator for the emetic act in dogs. Neurosci Res 14: $166-179$.
Lasiter PS, Glanzman DL (1982) Cortical substrates of taste aversion learning: dorsal prepiriform (insular) lesions disrupt taste aversion learning. J Comp Physiol Psychol 96:376-392.

Paxinos G, Watson C (1986) The rat brain in stereotaxic coordinates, 2nd ed. Orlando: Academic.

Reilly S, Grigson PS, Norgren R (1993) Parabrachial nucleus lesions and conditioned taste aversion: evidence supporting an associative deficit. Behav Neurosci 107:1005-1017.

Riley AL, Tuck DL (1985) Conditioned taste aversions: a behavioral index of toxicity. Ann NY Acad Sci 443:272-292.

Simbayi LC, Boakes RA, Burton MJ (1986) Effects of basolateral amygdala lesions on taste aversion produced by lactose and lithium chloride in the rat. Behav Neurosci 100:455-465.

Spector AC, Grill HJ (1986) Associative changes in taste reactivity during a lithium-induced internal state in intact and chronic decerebrate rats. Soc Neurosci Abstr 12:793.

Spector AC, Norgren R, Grill HJ (1992) Parabrachial gustatory lesions impair taste aversion learning in rats. Behav Neurosci 106:147-161.

Swank MW, Bernstein IL (1994) c-Fos induction in response to a conditioned stimulus after single trial taste aversion learning. Brain Res 636:202-208.

Swank MW, Schafe GE, Bernstein IL (1995) c-Fos induction in response to taste stimuli previously paired with amphetamine or $\mathrm{LiCl}$ during taste aversion learning. Brain Res 673:251-261.

Vangiersbergen PL, Palkovits M, DeJong W (1992) Involvement of neurotransmitters in the nucleus tractus solitarii in cardiovascular regulation. Physiol Rev 72:789-824.

Yamamoto R, Fujimoto Y (1991) Brain mechanisms of taste aversion learning in the rat. Brain Res Bull 27:403-406. 